\title{
CONHECIMENTO DOS PROFESSORES DO ENSINO FUNDAMENTAL QUANTO AO MANEJO EMERGENCIAL DE TRAUMATISMO DENTÁRIOS
}

\section{KNOWLEDGE OF PRIMARY SCHOOL TEACHERS IN EMERGENCY MANAGEMENT OF TOOTH INJURIES}

\author{
Hugo Pereira Vilela'; Carla Oliveira Favretto²; Talita Tartari³; Natália Galvão Garcia4 \\ 1 - Aluno de graduação do Curso de Odontologia - Faculdade de Morgana Potrich (FAMP). Mineiros - Goiás, Brasil. \\ 2 - Professora do Curso de Odontologia - Faculdade de Morgana Potrich (FAMP). Mineiros - Goiás, Brasil. \\ 3 - Pós-doutoranda do Programa de Pós-graduação da Faculdade de Odontologia de Bauru - Universidade de São. Bauru - São Paulo, Brasil. \\ 4 - Professora do Curso de Odontologia - Faculdade de Morgana Potrich (FAMP). Mineiros - Goiás, Brasil.
}

\section{RESUMO}

Objetivo: Avaliar o nível de conhecimento e segurança de professores do ensino fundamental para lidar com injúrias traumáticas na dentição permanente, antes e após assistirem uma palestra educativa. Materiais e Métodos: Os dados foram coletados por meio de questionários aplicados aos professores de ensino fundamental do $1^{\circ}$ ao $9^{\circ}$ ano do município de Mineiros-GO, antes e após assistirem uma palestra sobre traumatismo dentário. A amostra foi composta por 32 professores do ensino fundamental de escolas privadas e estaduais do município. Estes professores responderam a um questionário, o qual primeiramente avaliava o perfil do participante e em uma segunda parte avaliava o conhecimento do mesmo sobre traumatismo dentário. Os dados obtidos foram submetidos à uma análise estatística descritiva, para verificar a frequência das respostas de cada questão antes e após a realização da palestra de orientação. Resultados: Apesar da maioria dos professores apresentar um bom tempo de experiência profissional e ter pós-graduação, $80 \%$ dos participantes relataram sentir-se despreparados para agir diante de um trauma dentário. Após as orientações fornecidas nas palestras educativas foram observados resultados bastante positivos, aumentando significativamente, de $12,5 \%$ para $46,8 \%$, o número de participantes que se sentia preparado para socorrer um aluno com trauma dentário. Conclusão: A falta de conhecimento dos professores em relação a injúria dentária foi evidente. Desse modo, a realização de palestras educativas para esse público mostra ser de grande valia e imprescindível para o sucesso e longevidade do tratamento.

Palavras-chave: Traumatismos dentários; Conhecimento; Educação em saúde.

\section{INTRODUÇÃO}

As lesões dentárias traumáticas (LDTs) apresentam uma elevada prevalência, representando um dos mais graves problemas de saúde bucal ${ }^{1,2}$. Elas apresentam significativo impacto sobre a qualidade de vida da pessoa afetada, pois podem causar dor e resultar em danos funcionais, como dificuldades na fala e na mastigação, além de danos estéticos, que podem causar problemas emocionais e psicológicos, afetando a autoestima e o relacionamento social ${ }^{3,4}$.

Embora as LDTs possam acontecer em qualquer idade, estudos epidemiológicos mostram que a faixa etária mais comum está entre os 8-12 $\operatorname{anos}^{5}$ com predominância em indivíduos do sexo masculino ${ }^{3,5,6}$ e os ambientes em que ocorrem com maior frequência são em casa, seguido pela escola ${ }^{7-9}$. Pelo menos metade das crianças em idade escolar tem a possibilidade de sofrer traumatismos alvéolo dentários ${ }^{10}$, sendo a queda associada a brincadeiras e esportes a maior causa desses danos e o professor um dos principais responsáveis pelo primeiro atendimento prestado $^{5,11,12}$.

O grau de instrução de professores no que diz respeito ao manejo de crianças com LTDs foi avaliado em diversos estudos ${ }^{13-26}$.
Estes trabalhos mostraram, por meio de entrevistas, que a maioria dos educadores não receberam um treinamento ou orientações sobre traumatismo dentário antes ou após a sua formação ${ }^{13,14}$, apenas um pequeno número dos entrevistados saberia como agir em casos de LDTs e seria capaz de realizar um socorro adequado, a maioria deles não se sentia apta para prestar os cuidados emergenciais e colocaria o dente em uma situação de risco, culminando com um prognóstico desfavorável ${ }^{13-26}$. Nos estudos em que foi realizado um treinamento ou fornecido material didático a fim de capacitar os professores quanto a conduta a ser adota frente a uma LDT, os autores observaram que ao reaplicar o questionário ocorreu uma melhora significativa na escolha das respostas corretas quanto aos procedimentos a serem adotados, mostrando a importância desse tipo de ação nas escolas e até mesmo da inclusão desse assunto na matriz curricular dos cursos de licenciatura ${ }^{13,22}$.

Assim, o objetivo desse trabalho foi avaliar por meio de um questionário aplicado antes e após uma palestra educativa, o nível de conhecimento e segurança de professores do ensino fundamental do município de Mineiros-GO para em lidar com traumatismo de dentes permanentes. 


\section{MATERIAIS E MÉTODOS}

Este estudo foi submetido à apreciação do Comitê de Ética em Pesquisa em Seres Humanos do Centro Universitário de Santa Fé do Sul - UNIFUNEC, e aprovado pelo mesmo (CAAE: 08213519.8.0000.5428).

Esta pesquisa foi realizada em escolas de ensino fundamental do município de Mineiros-GO com professores de $1^{\circ}$ ao $9^{\circ}$ ano, após a solicitação, junto à Secretária de Educação do município e à direção das escolas.

O universo da pesquisa foi composto por professores que preencheram os critérios de inclusão, sendo eles: ser professor do ensino fundamental, estar presente na instituição de ensino no dia e hora agendados para a realização do estudo e da palestra educativa e aceitar participar da pesquisa por meio da assinatura do TCLE. Foram excluídos os que se negaram a participar da pesquisa ou que não estiveram presentes no momento do primeiro levantamento.

Para a coleta dos dados, foram agendados os dias nas escolas para aplicação dos questionários e realização da palestra educativa. O questionário utilizado nesta pesquisa foi auto aplicado em local reservado e sem conter informações que identificasse os participantes. Este era constituído por questões fechadas, com múltiplas escolhas, e semiabertas extraídas e adaptadas do estudo Espínola et al. ${ }^{13}(2017)$ sendo repassado para os professores após a explicação dos objetivos e metodologia da pesquisa e solicitação de assinatura do TCLE. O questionário foi dividido em duas partes: a primeira contendo perguntas quanto ao perfil do entrevistado como: sexo, idade, tempo de trabalho como professor e participação em cursos de primeiros socorros e traumatismo dentário. A segunda parte abordava questões referentes ao conhecimento dos entrevistados frente ao traumatismo dentário, possíveis experiências e condutas neste contexto, reimplante dentário, armazenamento e cuidados com o dente. As respostas dos participantes sobre as condutas foram avaliadas, e consideradas adequadas ou inadequadas, de acordo com as orientações

Tabela 1 - Caracterização sócio demográfica dos entrevistados

\begin{tabular}{|c|c|c|}
\hline Variáveis & & $\mathrm{N}(\%)$ \\
\hline \multirow{2}{*}{ Sexo } & Feminino & $26(81,2 \%)$ \\
\hline & Masculino & $6(18,8 \%)$ \\
\hline \multirow{5}{*}{ Idade } & $<20$ anos & - \\
\hline & 20-29 anos & $4(12,5 \%)$ \\
\hline & 30-39 anos & $11(34,4 \%)$ \\
\hline & 40-49 anos & $9(28,1 \%)$ \\
\hline & $>49$ anos & $8(25,0 \%)$ \\
\hline \multirow{8}{*}{ Experiência profissional } & $<01$ ano & - \\
\hline & 01-05 anos & $6(18,7 \%)$ \\
\hline & 06-10 anos & $2(6,3 \%)$ \\
\hline & $11-15$ anos & $6(18,7 \%)$ \\
\hline & $16-20$ anos & $9(28,1 \%)$ \\
\hline & $21-25$ anos & $3(9,4 \%)$ \\
\hline & $26-30$ anos & $3(9,4 \%)$ \\
\hline & $>30$ anos & $3(9,4 \%)$ \\
\hline \multirow{4}{*}{ Nível de formação } & Ensino Médio & - \\
\hline & Superior Incompleto & - \\
\hline & Superior Completo & $10(31,2 \%)$ \\
\hline & Pós-graduação & $22(68,8 \%)$ \\
\hline
\end{tabular}

clínicas preconizadas pela Associação Internacional de Trauma Dentário (AITD) ${ }^{2}$.

O referido questionário foi aplicado duas vezes: antes e após 7 dias que os professores assistiram uma palestra sobre traumatismo dentário ministrada por acadêmicos de Odontologia do último período, sob supervisão da professora coordenadora do projeto.

Além disso, os participantes também receberam um folder, adaptado do trabalho de $\operatorname{Ramos}^{1}$ (2014) contendo as orientações quanto ao protocolo para o atendimento emergencial de traumatismo dentário em escolas.

As respostas coletadas nos questionários foram tabuladas em uma planilha do Microsoft Excel (Microsoft Windows, 2010) formando um banco de dados. As variáveis quantitativas foram submetidas à estatística descritiva, verificando a frequência das respostas de cada questão antes e após a realização da palestra de orientação.

\section{RESULTADOS}

A amostra foi composta por 32 professores do ensino fundamental de duas escolas privadas e quatro escolas públicas do município de Mineiros-GO.

A tabela 1 apresenta a caracterização sócio demográfica dos entrevistados, na qual pode-se observar que os profissionais em sua maioria eram do sexo feminino $(81,2 \%)$, com idade abaixo de 49 anos (75,0\%). O tempo de experiência foi bastante variável, predominando entre $16-20$ anos (28,1\%). E o nível de formação mostrou que a maioria tinha pós-graduação $(68,8 \%)$.

$\mathrm{O}$ perfil dos entrevistados quanto ao traumatismo dentário também foi avaliado, tendo sido observado que apesar de grande parte dos participantes terem realizado curso de primeiros socorros durante ou fora da formação acadêmica, o tema "traumatismo dentário" não foi abordado em $81,2 \%$ dos casos. Desse modo, $100 \%$ dos entrevistados afirmaram que gostariam de receber informações sobre o tema (Figura 1).

Na segunda parte do questionário foi avaliado o conhecimento prévio dos participantes quanto ao traumatismo dentário em situações que podem ocorrer na rotina escolar. Ao serem questionados sobre o que fariam se uma criança quebrasse o dente da frente superior, a maior parte dos participantes afirmaram que levariam ou contactariam os pais para levá-la imediatamente ao

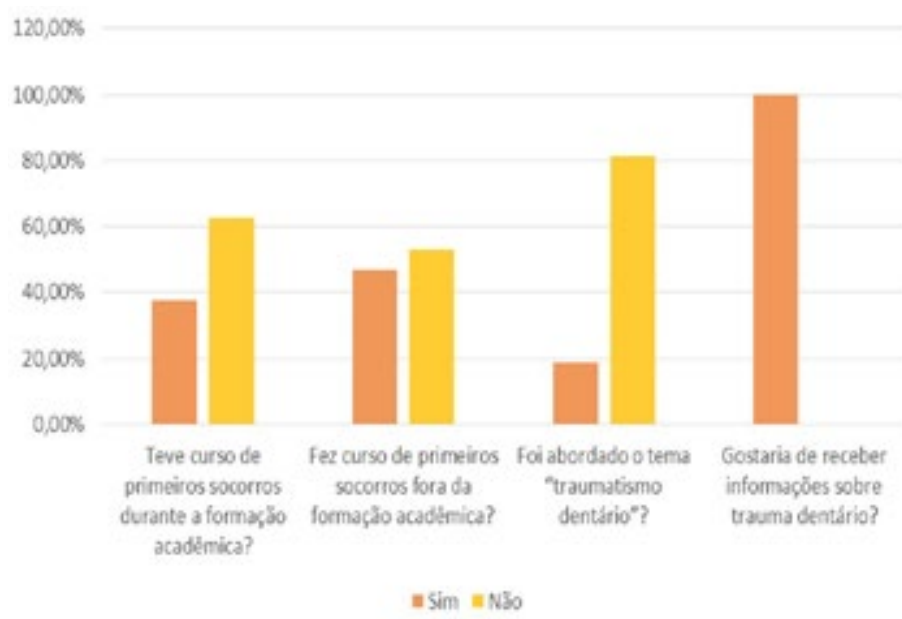

Figura 1 - Perfil dos entrevistados quanto ao tema "traumatismo dentário" 
dentista $(37,5 \%)$ ou ainda afirmaram que procurariam o fragmento do dente e em seguida levariam ou contactariam os pais para levá-la imediatamente ao dentista $(21,9 \%)$.

Os participantes também foram questionados sobre como agiriam ao encontrar um dente avulsionado de um aluno. E como pode ser observado na Figura 2, a maioria afirmou que pegaria o dente pela coroa $(53,1 \%)$ e nenhum dos participantes afirmou que pegaria o dente pela raiz.

Quanto ao tempo ideal para procurarem atendimento diante de um dente avulsionado, 78,1\% dos participantes responderam "imediatamente" e 21,9\% responderam "dentro de poucas horas".

A conduta dos entrevistados frente ao que deveria ser feito antes de recolocar o dente avulsionado no local também foi avaliada e a maioria dos entrevistados afirmou que enxaguaria o dente em água da torneira (62,5\%), enquanto que $25,0 \%$ afirmaram não saber o que fazer e $12,5 \%$ disseram que escovariam o dente suavemente com uma escova de dentes.

Considerando que a maioria dos participantes $(87,5 \%)$ afirmou que não recolocaria o dente no local, estes foram avaliados quanto ao acondicionamento do dente avulsionado. Na Tabela 2 pode-se observar que $34,4 \%$ dos entrevistados acondicionariam o dente em um pedaço de pano ou papel limpo, assim como a mesma porcentagem de participantes $(34,4 \%)$ responderam que acondicionariam em meio líquido. Ao serem questionados sobre qual líquido usariam, a maioria afirmou que acondicionaria em soro fisiológico (56,2\%) e em água da torneira (31,2\%) (Tabela 2).

Logo após responderem o questionário, os participantes receberam orientações sobre traumatismo dentário por meio de palestras e através de um folder contendo o protocolo para atendimento emergencial.

Sete dias depois, os mesmos participantes foram convidados a responderem novamente ao questionário.

Ao comparar a segurança dos entrevistados frente a um traumatismo dentário antes e depois das orientações recebidas, pôde-se observar que o número de participantes que se sentia preparado para socorrer um aluno com trauma dentário aumentou depois do recebimento das orientações. Assim como, aumentou o número e participantes que recolocaria o dente no local (Figura 3).

Além disso, quando comparadas as respostas dos questionários antes e depois das orientações foram observadas melhorias quanto à conduta dos participantes frente ao traumatismo dentário.

A Figura 4 apresenta uma comparação do conhecimento dos entrevistados quanto ao acondicionamento de um dente avulsionado antes e depois das orientações fornecidas. Podendo ser observado um resultado positivo, ao ser identificado que aumentou o número de participantes que acondicionariam o dente em líquido e na boca, assim como também diminuiu o acondicionamento em gelo, pedaço de pano ou papel e em saco plástico.

\section{DISCUSSÃO}

Ambientes escolares concentram muitas crianças envolvidas em atividades recreativas. Em virtude disso, episódios de traumatismos são frequentes, sendo a cavidade bucal muitas vezes acometida nesses acidentes. Desse modo, o preparo dos professores no manejo emergencial das crianças que sofrem
Tabela 2 - Conhecimento dos entrevistados quanto ao acondicionamento do dente avulsionado.

\begin{tabular}{|c|c|c|}
\hline Questões & Alternativas & $\mathrm{N}(\%)$ \\
\hline \multirow{7}{*}{$\begin{array}{l}\text { Se você não reco- } \\
\text { locasse o dente no } \\
\text { lugar, como você o } \\
\text { acondicionaria para } \\
\text { que fosse levado ao } \\
\text { dentista? }\end{array}$} & Gelo & $5(15,5 \%)$ \\
\hline & Líquido & $11(34,4 \%)$ \\
\hline & Na boca do aluno & $2(6,3 \%)$ \\
\hline & Na mão & - \\
\hline & Em um pedaço de pano ou papel limpo & $11(34,4 \%)$ \\
\hline & Em um saco ou recipiente de plástico & $3(9,4 \%)$ \\
\hline & $\begin{array}{l}\text { Jogaria o dente no lixo apropriado para } \\
\text { evitar contaminação e infecções }\end{array}$ & - \\
\hline \multirow{6}{*}{$\begin{array}{l}\text { Se você utilizas- } \\
\text { se líquido para } \\
\text { levar o dente, qual } \\
\text { escolheria? }\end{array}$} & Água da torneira & $10(31,2 \%)$ \\
\hline & Leite fresco & $2(6,3 \%)$ \\
\hline & Álcool & - \\
\hline & Água da geladeira & $2(6,3 \%)$ \\
\hline & Soro fisiológico & $18(56,2 \%)$ \\
\hline & Solução antisséptica & - \\
\hline
\end{tabular}

Qual seria sua conduta ao encontrar um dente avulsionado de um aluno?

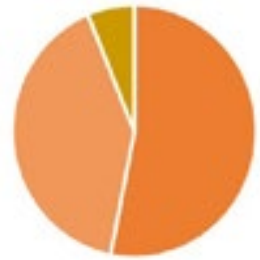

a Pegaria o dente pela coroa

- Pegaria o dente pela raiz

= Pegaria o dente em qualquer posição

- Não pegaria o dente

Figura 2 - Conduta dos entrevistados frente a um dente avulsionado

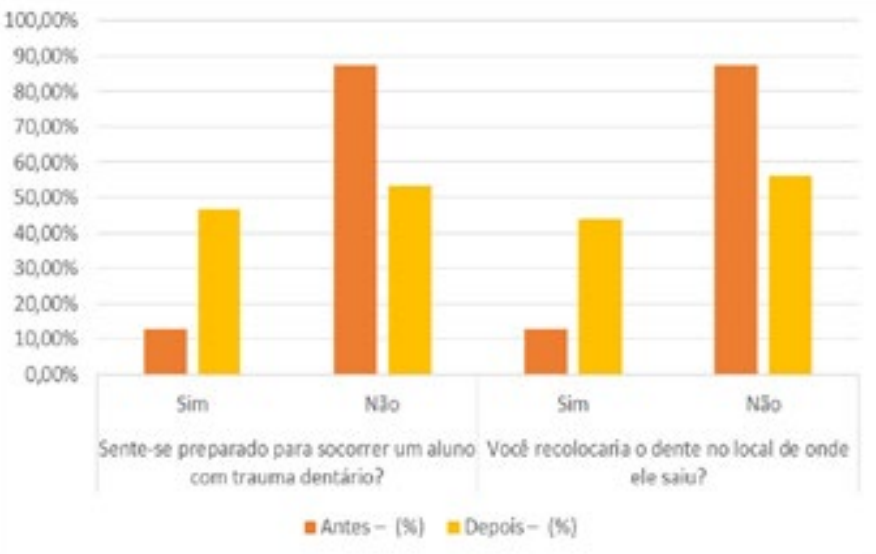

Figura 3 - Comparação quanto à segurança dos entrevistados frente a um traumatismo dentário antes e depois das orientações recebidas

\section{Como você acondicionaria o dente avulsionado?}

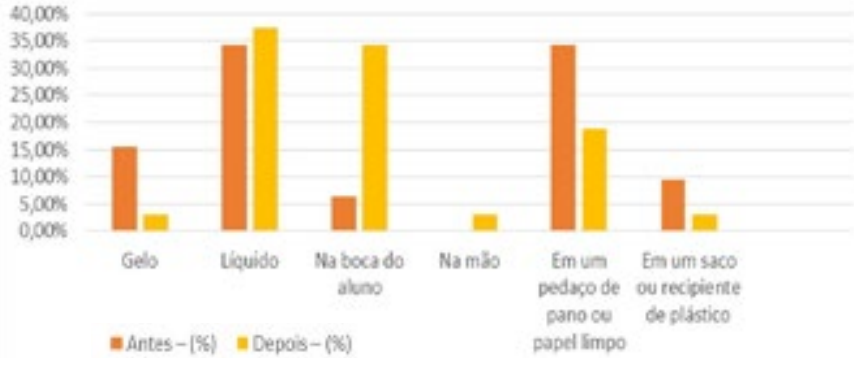

Figura 4 - Comparação do acondicionamento do dente avulsionado antes e depois das informações recebidas 
traumatismo dentário é fundamental para o sucesso e longevidade do tratamento ${ }^{17}$. Entretanto, a literatura mostra que estes profissionais, na maioria das vezes, não estão preparados para agir nessas situações devido à falta de conhecimento sobre o assunto $^{21}$.

O presente estudo foi constituído por uma amostra de professores com um bom tempo de experiência profissional, e em sua maioria, pós-graduados. No entanto, apesar de grande parte dos participantes terem realizado curso de primeiros socorros durante ou fora da formação acadêmica, $80 \%$ dos professores relataram sentir-se despreparados para agir diante de um trauma dentário. Havendo o interesse de $100 \%$ dos entrevistados em receber informações sobre o tema. Corroborando com esses resultados, um estudo realizado com um grupo de professores em Hong Kong mostrou que estes também apresentavam deficiência em procedimentos de urgência frente a traumatismos dentários, estando apenas $4 \%$ dos participantes preparados ${ }^{23}$.

Além disso foi observado que na maioria das vezes, os cursos de primeiros socorros não abordavam o tema traumatismo dentário. Segundo Silva et al. ${ }^{24}$ (2009), após realizarem uma avaliação em uma creche, observaram que apesar de $26,7 \%$ dos professores da equipe possuir uma capacitação de primeiros socorros, apenas 3,3\% receberam orientações sobre traumatismo dentário.

$\mathrm{Na}$ segunda parte do questionário realizado no presente estudo, foi avaliado o conhecimento prévio dos participantes quanto ao traumatismo dentário em situações que podem ocorrer na rotina escolar. Logo, os participantes foram questionados sobre a conduta, tempo e armazenamento. E os resultados obtidos indicaram que a maioria dos professores apresentava conhecimento aquém do necessário para agir diante de um trauma dentário, assim como tem sido apontado por diversos estudos da literatura atual ${ }^{13,17,25,26}$.

Essa falta de capacitação dos professores reflete diretamente no prognóstico, pois este está relacionado a conduta, ao tempo decorrido e ao local de armazenamento, caso o dente não seja recolocado imediatamente. Desse modo, muitos autores acreditam que a realização de palestras educativas para esse público possa ser de grande valia ${ }^{13,17,25,26}$.

No presente estudo após as orientações fornecidas na palestra educativa foram observados resultados bastante positivos. Como por exemplo, ao comparar a segurança dos entrevistados frente a um traumatismo dentário antes e depois das orientações recebidas, pode-se observar que o número de participantes que se sentia preparado para socorrer um aluno com trauma dentário aumentou depois do recebimento das orientações. Assim como, aumentou o número e participantes que recolocaria o dente no local.

Também foi notória a contribuição para melhorias significativas quanto à conduta dos participantes frente ao traumatismo dentário, aumentando significativamente o número de participantes que acondicionariam o dente em líquido e na boca, e diminuindo o acondicionamento em gelo, pedaço de pano ou papel e em saco plástico.

Diante disso, fica claro que o conhecimento e aplicação do protocolo adequado de atendimento emergencial para traumatismo dentário é imprescindível para o sucesso e longevidade do tratamento.

\section{CONCLUSÃO}

A falta de conhecimento dos professores em relação ao trauma dental foi evidente. Desse modo, a realização de palestras educativas para esse público foi de grande valia, aumentando significativamente o número de participantes que se sentia preparado para socorrer um aluno com traumatismo dentário e consequentemente favorecendo o sucesso e longevidade do tratamento.

\section{REFERÊNCIAS}

01. Ramos TS. Complementação do protocolo para o suporte básico de vida do CBMGO. Goiânia Academia Bombeiro Militar; 2014.

02. Andreasen J, Andreasen F, Bakland L, Flores M. Injuries to the primary dentition. Traumatic dental injuries - a manual Copenhagen: Munksgaard. 1999:44-7.

03. Traebert JL. Traumatismo dentário: um estudo de caso-controle de base populacional em escolares de 11 a 13 anos de idade e suas famílias. [Tese de Doutorado] Florianópolis: Universidade Federal de Santa Catarina; 2002

04. Marcenes W, Zabot NE, Traebert J. Socio-economic correlates of traumatic injuries to the permanent incisors in schoolchildren aged 12 years in Blumenau, Brazil. Dent Traumatol. 2001 Oct;17(5):222-6.

05. Bastone EB, Freer TJ, McNamara JR. Epidemiology of dental trauma: a review of the literature. Aust Dent J. 2000 Mar;45(1):2-9.

06. de Leão BLC, Lima C, Neto JS, Perin CP, Mattos NHR. Nível de conhecimento sobre o pronto atendimento ao traumatismo alvéolo dentário e aquisição de conhecimento por meio de leitura de panfleto educativo. Revista da Faculdade de Odontologia-UPF. 2017;22(2):172-6.

07. Costa LED, Queiroz FdS, Nóbrega CBC, Leite MS, Nóbrega WFS, Almeida ERd. Trauma dentário na infância: avaliação da conduta dos educadores de creches públicas de Patos-PB. Rev odontol UNESP. 2014;43(6):402-8.

08. Skaare AB, Jacobsen I. Primary tooth injuries in Norwegian children (1-8 years). Dent Traumatol. 2005 Dec;21(6):315-9.

09. García Godoy F, Sánchez R, Sánchez JR. Traumatic dental injuries in a sample of Dominican schoolchildren. Community dentistry and oral epidemiology. 1981;9(4):193-7.

10. Andreasen JO, Ravn JJ. Epidemiology of traumatic dental injuries to primary and permanent teeth in a Danish population sample. Int J Oral Surg. 1972;1(5):235-9.

11. Skaare $A B$, Jacobsen I. Etiological factors related to dental injuries in Norwegians aged 7-18 years. Dent Traumatol. 2003 Dec;19(6):304-8.

12. Granville-Garcia AF, Lima EM, Gomes Santos P, de Menezes VA. Avaliação do conhecimento dos professores de educação física de Caruaru-PE sobre avulsão-reimplante. Pesquisa Brasileira em Odontopediatria e Clínica Integrada. 2007;7(1):15 - 20

13. Espínola WdC, Rodrigues HB, Ribeiro JAA, Lopes JN, Pinheiro SAdA. Conhecimento dos professores de creches e escolas sobre traumatismos dentários Temas em Saúde. 2017;17(2):39-60.

14. Çaglar E, Ferreira LP, Kargul B. Dental trauma management knowledge among a group of teachers in two south European cities. Dental Traumatology. 2005;21(5):258-62.

15. Campos MIC, Henriques KAM, Campos CN. Nível de informação sobre a conduta de urgência frente ao traumatismo dental com avulsão. Pesquisa Brasileira em Odontopediatria e Clínica Integrada. 2006;6(2).

16. Curylofo PA, Lorencetti KT, da Silva SRC. Avaliação do conhecimento de professores sobre avulsão dentária. Arquivos em Odontologia. 2012;48(3):175-80. 
17. Bittencourt AM, Pessoa OF, Silva J. Avaliação do conhecimento de professores em relação ao manejo da avulsão dentária em crianças. Rev Odontol UNESP. 2008;37(1):15-9.

18. Pacheco LF, Filho PFG, Letra A, Menezes R, Villoria GEM, Ferreira $\mathrm{SM}$. Evaluation of the knowledge of the treatment of avulsions in elementary school teachers in Rio de Janeiro, Brazil. Dental Traumatology. 2003;19(2):76-8.

19. Costa AdBMd. Traumatismos alvéolo-dentários: avaliação dos conhecimentos e atitudes de uma amostra de Professores do Ensino Fundamental do Município de São Paulo.[Dissertação de Mestrado]. São Paulo: Universidade de São Paulo; 2004.

20. Mori GG, Turcio KHL, Borro VPB, Mariusso ÂM. Evaluation of the knowledge of tooth avulsion of school professionals from Adamantina, São Paulo, Brazil. Dental Traumatology. 2007;23(1):2-5.

21. Blakytny C, Surbuts C, Thomas A, Hunter M. Avulsed permanent incisors: knowledge and attitudes of primary school teachers with regard to emergency management. International journal of paediatric dentistry. 2001;11(5):327-32.
22. Moraes FdM. Avulsão dentária: características gerais, meios de armazenamento e protocolos terapêuticos. [Trabalho de Conclusão de Curso]. Piracicaba: Faculdade de Odontologia de Piracicaba da Universidade Estadual de Campinas; 2014.

23. Chan AWK, Wong TKS, Cheung GSP. Lay knowledge of physical education teachers about the emergency management of dental trauma in Hong Kong. Dent Traumatol 2001; 17:77-85.

24. Silva MB, Costa AMM, Almeida MEC, Maia AS, Carvalhal CIO, Resende GB. Avaliação do conhecimento da abordagem de trauma dental, pelos profissionais de creches. Conscientia e Saúde. 2009; 8(1): 65-73.

25. Berti M, Furlanetto DLC, Refosco MZ. Avaliação do Conhecimento de Professores do Ensino Fundamental sobre o Tema Avulsão Dentária Pesquisa Brasileira em Odontopediatria e Clínica Integrada. 2011; 11(3): 381-386.

26. Ribeiro RAO, Souza DFS, Souza FV, Teixeira HM, Nascimento ABL. Avaliação do Conhecimento de Profissionais frente ao Trauma Dental em Crianças do Ensino Fundamental em Pernambuco. Odontol. Clín.-Cient. 2017; 16(3) 179- 184.

\section{ABSTRACT}

Objective: To evaluate the level of knowledge and safety of primary school teachers in dealing with traumatic injuries in permanent teeth, before and after attending an educational lecture. Materials and methods: Data were collected through questionnaires applied to primary school teachers from the $1^{\text {st }}$ to the $9^{\text {th }}$ year of the city of Mineiros-GO, before and after attending a lecture on dental injuries. The sample consisted of 32 school teachers from private and state schools. These teachers answered a questionnaire, which first evaluated the participant's profile and in a second part evaluated their knowledge about tooth injuries. The data were submitted to a descriptive statistical analysis, to verify the frequency of the answers of each question before and after the orientation lecture. Results: Even though most teachers have a good amount of professional experience and have a postgraduate degree, $80 \%$ of the participants reported feeling unprepared to act in the face of tooth injuries. Following the guidelines provided in the educational lectures were observed quite positive results, significantly increasing, of $12.5 \%$ to $46.8 \%$, the number of participants who felt prepared to succor a student with tooth injuries. Conclusion: The teachers' lack of knowledge about dental injuries was evident. Thus, educational lectures for this audience showed to be of great value and essential for the success and longevity of the treatment.

Keywords: Tooth Injuries. Knowledge. Health Education.

\section{AUTOR DE CORRESPONDÊNCIA:}

Natália Galvão Garcia natggalvao@hotmail.com

Faculdade de Morgana Potrich (FAMP).

Av. Antônio Carlos Paniago, 65, Setor Mundinho - CEP

75832-005 - Mineiros-Goiás, Brasil

(31) 994033999 